\title{
Prospective clinical and manometric study comparing pneumatic dilatation and sublingual nifedipine in the treatment of oesophageal achalasia
}

\author{
G Coccia, M Bortolotti, P Michetti, M Dodero
}

\begin{abstract}
A study was carried out in 30 patients affected by a mild or moderate degree of oesophageal achalasia to compare the clinical and manometric effects of sublingual nifedipine and pneumatic dilatation. Sixteen patients were dilated twice with Rider-Moeller dilators and 14 were treated with sublingual nifedipine 10$20 \mathrm{mg} 30$ minutes before meals. A manometric evaluation was performed before and six months after starting treatment. The clinical evaluation (according to Vantrappen's criteria) was performed every three months for a mean follow up of 21 months. In both groups of patients a significant $(p<0.001)$ fall in lower oesophageal sphincter pressure was observed after treatment and excellent or good clinical results were observed in $75 \%$ of dilated patients and in $77 \%$ of patients treated with nifedipine. One patient could not tolerate nifedipine. No complications were observed after dilatation. It is concluded that longterm treatment with sublingual nifedipine and pneumatic dilatation are equally effective in the treatment of oesophageal achalasia of mild or moderate degree.
\end{abstract}

The traditional approach to the treatment of patients with achalasia is based on stretching or cutting the muscle fibres at the oesophagogastric junction by pneumatic dilatation ${ }^{1-6}$ or surgical myotomy..$^{7-12}$ More recently, nitro derivatives and calcium antagonists, particularly nifedipine, have been proved to be effective in reducing lower oesophageal pressure and improving oesophageal emptying in patients with achalasia. ${ }^{13-17}$ The results obtained both with surgical or mechanical procedures, such as myotomy and dilatation, and with drug treatment are well known. ${ }^{18-20}$ No prospective study, however, has been reported comparing drug treatment with nifedipine and pneumatic dilatation.

The aim of our study was to perform such a prospective trial to determine whether there is a significant difference in the results of both treatments.

\section{Methods}

Thirty consecutive patients with clinical, radiological, endoscopic, and manometric evidence of oesophageal achalasia, stage I or II using the classification of Adams et $a l,{ }^{21}$ were randomly assigned to two groups. Patients with sigmoid dilatation of the oesophagus or those who had had treatment for achalasia were excluded. Group A had 16 patients assigned to undergo pneumatic dilatation (seven men, nine women; mean age 49.5 years, range 18-82 years). The mean duration of symptoms was $4 \cdot 1$ years (range 6 months -8 years) and the mean oesophageal diameter $3.8 \mathrm{~cm}$ (range $2-6 \mathrm{~cm}$ ). Group B had 14 patients who were treated with sublingual nifedipine (six men, eight women; mean age 51.3 years, range $17-83$ years). The mean duration of symptoms was 3.5 years (range 6 months -7 years) and the mean oesophageal diameter $3.9 \mathrm{~cm}$ (range $2 \cdot 5-6 \mathrm{~cm}$ ).

\section{CLINICAL STUDY}

The patients were examined and details of the severity of dysphagia, regurgitation, retrosternal pain, and weight loss were taken before the study and every three months for a mean follow up of 21.4 months (range 12-36, median 21 months) according to the criteria of Vantrappen and Hellemans. ${ }^{2}$ Patients were classified on the basis of the results of each treatment: excellent, good, moderate, and poor.

\section{MANOMETRIC STUDY}

Manometry was performed with a polyvinyl probe with five orifices, three of which, radially orientated, were positioned at the level of the lower oesophageal sphincter and two in the oesophageal body 5 and $10 \mathrm{~cm}$ above the sphincter. Each catheter was perfused with distilled water by a pneumohydraulic pump and connected to Statham P $23 \mathrm{Db}$ transducers and a multichannel polygraph. The compliance of our system showed a rise in pressure of $150 \mathrm{mmHg} / \mathrm{s}$ on occlusion. After the patients had fasted overnight the probe was passed through the nose into the stomach and the lower oesophageal sphincter pressure was evaluated with a slow gastro-oesophageal pullthrough. Pressure was expressed as the mid-inspiratory pressure using intragastric pressure as zero reference. In group B patients, after a recording period of at least 30 minutes nifedipine $20 \mathrm{mg}$ was administered sublingually and pressure recorded for another 45 minutes. In all patients a 
manometric control was performed six months after dilatation in group $A$ and after six months of treatment in group $B$.

\section{TREATMENT}

In patients in group $A$, after an overnight fast and premedication with intravenous benzodiazepines a Rider-Moeller pneumatic dilator (35 mm diameter) was positioned at the oesophagogastric junction over a guide wire and rapidly inflated to $200 \mathrm{mmHg}$ for one minute and then to $300 \mathrm{mmHg}$ for another minute. ${ }^{2}$ The procedure was repeated 48 hours later with a larger dilator (40 $\mathrm{mm}$ diameter). After each dilatation an endoscopic control was performed to exclude complications such as perforation or haemorrhage. All patients were treated as outpatients.

In patients in group B nifedipine $10-20 \mathrm{mg}$ was administered sublingually 30 minutes before each meal up to a maximum dose of $80 \mathrm{mg} /$ day (mean $55 \mathrm{mg} /$ day). The dose was chosen after a trial lasting a week.

Manometric results were expressed as mean (SD). Student's $t$ test for paired and unpaired data and Wilcoxon's rank sum test were used for statistical evaluation.

\section{Results}

\section{CLINICAL STUDY}

Before treatment all patients had had dysphagia and regurgitation daily. In group A excellent or good results were obtained in 12 patients, moderate results in two, and poor results in two. No complications were observed in this group. In group B excellent or good results were obtained in 10 patients, moderate results in two, and poor results in one. One patient could not tolerate nifedipine because of headache. No patient developed a tolerance for nifedipine during follow up.

\section{MANOMETRIC RESULTS}

In group $A$ patients the mean (SD) lower oesophageal sphincter pressure before treatment was 40.9 (15) $\mathrm{mmHg}$ and after treatment the pressure fell to $20.5(7) \mathrm{mmHg}(\mathrm{p}<0.001)$. In group B the mean lower oesophageal sphincter pressure before treatment was 40.8 (11) $\mathrm{mmHg}$ and 30 minutes after nifedipine administration it fell to 22.5 (8) $\mathrm{mmHg}(\mathrm{p}<0.001)$. After six months of treatment it was $25 \cdot 7$ (6) $\mathrm{mmHg}$ $(\mathrm{p}<0.001)$.

\section{Discussion}

In our study both pneumatic dilatation and treatment with sublingual nifedipine reduced lower oesophageal sphincter pressure in patients with achalasia by $50 \%$ and $48 \%$, respectively, from the pretreatment value. This result is in agreement with previous reports which showed a decrease in lower oesophageal sphincter pressure ranging from $42 \%$ to $51 \%$ in association with clinical improvement. ${ }^{12225}$ Clinical follow up, even when short, showed that both treatments are effective in reducing symptoms and that excellent or good results are obtained in nearly the same proportions of patients. Our results confirm that pneumatic dilatation is a rapid procedure requiring no hospitalisation and in our patients no immediate complications were observed. There is, however, some risk of perforation, haemorrhage, or subsequent gastrooesophageal reflux. ${ }^{26}$ Moreover, some rupture of the mucosa often occurs, with fibrosis causing problems if surgery is subsequently performed. ${ }^{23}$

On the other hand, sublingual nifedipine is unpleasant ${ }^{27}$ and in some patients can cause excessive vasodilatation with peripheral oedema, headache, and hypotension. But only one of our patients was unable to tolerate nifedipine because of headaches and no hypotension occurred, even at doses up to $80 \mathrm{mg} /$ day. Finally, in no case did tolerance to the drug develop.

Our manometric and clinical results are in agreement with previous studies. In fact Traube et al obtained a significant decrease in lower oesophageal sphincter pressure after $10 \mathrm{mg}$ of sublingual nifedipine, ${ }^{19}$ and Gelfond et al, who administered oral nifedipine, with a consequent delay and decrease in drug adsorption, obtained satisfactory results. ${ }^{27}$ Our results, however, contrast with a recent report from Robertson et $a l$, who obtained no appreciable decrease in lower oesophageal sphincter pressure after nifedipine administration. ${ }^{25}$ The methods of that trial are questionable because the lower oesophageal sphincter pressure was measured only 15 minutes after nifedipine was given and was compared with a value measured in a previous manometric examination. It is known that nifedipine has its maximum effect on lower oesophageal sphincter pressure 30 minutes after sublingual administration..$^{28}$ Finally, regarding the clinical part of that study, unfortunately nifedipine was given at too low a dose $(10 \mathrm{mg})$, at too short intervals before meals ( 15 minutes), and to a population of unselected patients, including those with sigmoid dilatation of the oesophagus.

The results of our study show that, provided that nifedipine is administered sublingually to patients with stage I or II achalasia at least 30 minutes before meals and in an individualised dose, sublingual nifedipine is as effective as pneumatic dilatation in the treatment of oesophageal achalasia.

1 Schindler R. Observations on cardiospasm and its treatmen by brusque dilatation. Ann Intern Med 1956; 45: 207-15.

2 Vantrappen G, Hellemans J. Treatment of achalasia and related motor disorders. Gastroenterology 1980; 79: 144-54.

3 Fellows JW, Ogilvie AL, Atkinson M. Pneumatic dilatation in achalasia. Gut 1983; 24: 1020-3.

4 Frimberger E, Kuhner W, Kuhnert H, Ottenjann R. Results of treatment with the endoscope dilator in 11 patients with achalasia of the esophagus. Endoscopy 1981; 13: 173-5.

5 Witzel L. Treatment of achalasia with a pneumatic dilator attached to a gastroscope. Endoscopy 1981; 13: 176-7.

$6 \mathrm{Cox} \mathrm{J}$, Buckton GK, Bennett JR. Balloon dilatation in achalasia: a new dilator. Gut 1986; $27: 986-9$.

7 Effler DB, Loop FD, Groves LK, Favaloro RG. Primary surgical treatment for esophageal achalasia. Surg Gynecol Obstet 1971; 132: 1057-63.

8 Menguy R. Management of achalasia by transabdominal cardiomyotomy and funduplication. Surg Gynecol Obste 1971; 133: 482-4.

9 Sariyannis C, Mullard KS. Oesophagomyotomy for achalasia of the cardia. Thorax 1975; 30: 539-42.

10 Jara FM, Toledo Pereyra LH, Lewis JW, Magilligan DJ. Long term results of esophagomyotomy for achalasia of the esophagus. Arch Surg 1979; 114: 935-6.

11 Black J, Vorbach AN, Collis JL. Results of Heller's operation 
of achalasia of the oesophagus. The importance of hiatal repair. Br 7 Surg 1976; 63: 949-53.

12 Donahue PE, Schlesinger PK, Bombeck CT. Achalasia of the esophagus. Treatment controversies and the method of choice. Ann Surg 1986; 203: 505-11.

13 Kikendall JW, Mellow HM. Effect of sublingual nitroglycerin and long acting nitrate preparations on esophageal motility. Gastroenterology 1980; 79: 703-6.

14 Rozen P, Gelfond M, Salzman S. Radionuclide confirmation of the therapeutic value of isosorbide dinitrate in relieving the dysphagia in achalasia. 7 Clin Gastroenterol 1981; 4: 17-22.

15 Bortolotti M. Labo'G. Clinical and manometric effects of nifedipine in patients with esophageal achalasia. Gastroenterology $1981 ; 80$ : 39-44. 16 Becker $\mathrm{BJ}$, Buraks

Becker BJ, Burakoff $R$. The effect of verapamil on the esophageal sphincter pressure in normal subjects and in achalasia. Am $\mathcal{F}$ Gastroenterol 1983; 78: 773-5.

17 Castell DO. Calcium channel blocking agents for gastrointestinal disorders. Am F Cardiol 1985; 55: 210B-3B.

18 Csendes A, Braghetto I, Henriquez A, Cortes C. Late results of a prospective randomized study comparing forceful dilatation and oesophagomyotomy in patients with achalasia. $G u$ 1989; 30: 299-304.

19 Traube M, Dubovik S, Magyar L, McCallum RW. Sublingual nifedipine in achalasia. A randomized double blind study. Gastroenterology 1986; 90: 1670.
20 Bennett JR. Treatment of achalasia: a review. $\mathcal{F} R$ Soc $M e d$ 1980; 73: 649-54.

21 Adams CWM, Brain RHF, Ellis FG. Achalasia of the cardia. Guy's Hospital Reports 1961; 110: 191-236.

22 Cohen S, Lipshutz W. Lower esophageal sphincter dysfunction in achalasia. Gastroenterology 1971; 61: 814-20.

23 Csendes A, Velasco N, Braghetto I, Henriquez A. A prospective randomized study comparing forceful dilatation and esophagomyotomy in patients with achalasia of the esophagomyotomy in patients with ach
esophagus. Gastroenterology 1981; 80: 789-95.

24 Lamet M, Fleshler B, Achkar E. Return of peristalsis in achalasia after pneumatic dilatation. Am $\mathcal{F}$ Gastroenterol 1985; 80: 602-4.

25 Robertson CS, Hardy JG, Atkinson M. Quantitative assessment of the response to therapy in achalasia of the cardia. Gut 1989; 30: 768-73.

26 Vantrappen G, Janssens J. To dilate or to operate? That is the question. Gut 1983; 24: 1013-9.

27 Gelfond M, Rozen P, Gilat T. Isosorbide dinitrate and nifedipine treatment of achalasia: a clinical, manometric and radionuclide evaluation. Gastroenterology 1982; 83: 963-9.

28 Hongo M, Traube M, McAllister RG, McCallum RW. Effects of nifedipine on esophageal motor function in humans: correlation with plasma nifedipine concentrations. Gastroenterology 1984; 86: 8-12. 